\title{
Radio frequency plasma processing effects on the emission characteristics of a MeV electron beam cathode
}

\author{
J. I. Rintamaki, ${ }^{\text {a) }}$ R. M. Gilgenbach, ${ }^{\text {b) }}$ W. E. Cohen, and R. L. Jaynes \\ Nuclear Engineering and Radiological Sciences Department, University of Michigan, Ann Arbor, \\ Michigan 48109
}

\author{
M. E. Cuneo and P. R. Menge \\ Sandia National Laboratories, Albuquerque, New Mexico 87185-1193
}

(Received 1 April 1999; accepted for publication 11 May 1999)

\begin{abstract}
Experiments have proven that surface contaminants on the cathode of an electron beam diode influence electron emission current and impedance collapse. This letter reports on an investigation to reduce parasitic cathode current loss and to increase high voltage hold off capabilities by reactive sputter cleaning of contaminants. Experiments have characterized effective radio frequency (rf) plasma processing protocols for high voltage anode-cathode (A-K) gaps using a two-stage argon/ oxygen and argon rf plasma discharge. Time-resolved optical emission spectroscopy measures contaminant (hydrogen) and bulk cathode (aluminum) plasma emission versus transported axial electron beam current turn on. Experiments were performed at accelerator parameters: $V=-0.7$ to $-1.1 \mathrm{MV}, I($ diode $)=3-30 \mathrm{kA}$, and pulse length $=0.4-1.0 \mu \mathrm{s}$. Experiments using a two-stage low power $(100 \mathrm{~W})$ argon/oxygen $\mathrm{rf}$ discharge followed by a higher power $(200 \mathrm{~W})$ pure argon rf discharge yielded an increase in cathode turn-on voltage required for axial current emission from $662 \pm 174 \mathrm{kV}$ to $981 \pm 97 \mathrm{kV}$. The turn-on time of axial current was increased from $100 \pm 22$ to $175 \pm 42$ ns. (C) 1999 American Institute of Physics. [S0003-6951(99)01827-6]
\end{abstract}

The theoretical electron field emission threshold for most metals is an applied surface electric field of order $10^{8} \mathrm{~V} / \mathrm{cm}$. The observed electron emission threshold is usually two or three orders of magnitude lower; an applied electric field of $10^{5}-10^{6} \mathrm{~V} / \mathrm{cm}$ is sufficient for electron emission from a cold metal cathode. This is believed to be due to the complex nature of the metal surface, which is composed of microprotrusions, metal oxide layers, and adsorbed surface contaminants. Previous work has established that the plasma layer formed on the cathodes of pulsed vacuum diodes largely determines many aspects of the performance of these devices. ${ }^{1-11}$ For example, the pulse length and current density are controlled by the expansion of plasmas formed from contaminants present on the anode and cathode surface. Thin layers of vacuum pump oil and water adsorbed onto vacuum surfaces exist in most practical vacuum systems. When these impurities dominate cathode plasma formation, variations in cathode material surface preparation have little effect on the cathode plasma properties. ${ }^{3}$ Experiments conducted at Sandia National Laboratories confirmed that surface contamination of anode-cathode surfaces from water vapor and hydrocarbons was a major concern for light ion beam fusion research., ${ }^{7,12}$ These contaminants are also the main source of parasitic current loss in ion diodes. Since water vapor readily adsorbs onto all vacuum surfaces open to air, most vacuum surfaces are coated with several monolayers of low- $Z(\mathrm{H})$ contamination. When a voltage is applied to an anodecathode gap, these monolayers of hydrogen-based contami-

\footnotetext{
a) Permanent address: GE Lighting, 1975 Noble Rd., Cleveland, OH, 44112.

${ }^{b)}$ Electronic mail: rongilg@umich.edu
}

nation break down into anode and cathode plasmas which travel across the $\mathrm{A}-\mathrm{K}$ gap, resulting in plasma closure and shorting of the diode.

In this letter we report an in situ method to raise the breakdown voltage limit of broad-area cathodes. Radio frequency (rf) plasma discharges of argon and argon/oxygen gas mixtures are used to reduce the level of surface contaminants and subsequently reduce parasitic current loss. ${ }^{7,12}$ The argon is used to sputter contaminants off of anode-cathode $(\mathrm{A}-\mathrm{K})$ surfaces, while the oxygen chemically sputters carbon and hydrogen from these surfaces to form carbon dioxide and water vapor that can then be pumped from the system. This work is a systematic study and optimization of $\mathrm{rf}$ plasma discharges for the control of cathode plasma formation in a high-power electron beam diode. This work directly establishes a strong link between breakdown limits and surface contamination in pulsed systems leading to cathode plasma formation, that have been suggested by previous work, based on indirect evidence.

Figure 1 is a diagram of the Michigan Electron Long Beam Accelerator (MELBA) vacuum chamber, showing schematically the A-K gap geometry. MELBA operating parameters are: $V=-0.7$ to $-1.1 \mathrm{MV}, I($ diode $)=3-30 \mathrm{kA}$, and pulse length $=0.4-1.0 \mu \mathrm{s}$. The anode is a titanium-6-4 disk inside a 304 stainless steel anode ring holder, while the cathode is a 4.1-cm-diam T3-2011 aluminum disk inserted into the center of a 304 stainless steel cathode holder. At a standard $4 \mathrm{~cm}$ A-K gap, MELBA has an average macroscopic electric field strength of $250 \mathrm{kV} / \mathrm{cm}$ at the aluminum cathode surface. The stainless steel cathode holder surfaces were micropolished. The anode was the powered electrode for the rf discharge. The fill gas was let in at a few mTorr pressure and the plasma would be ignited for 5-30 min, 


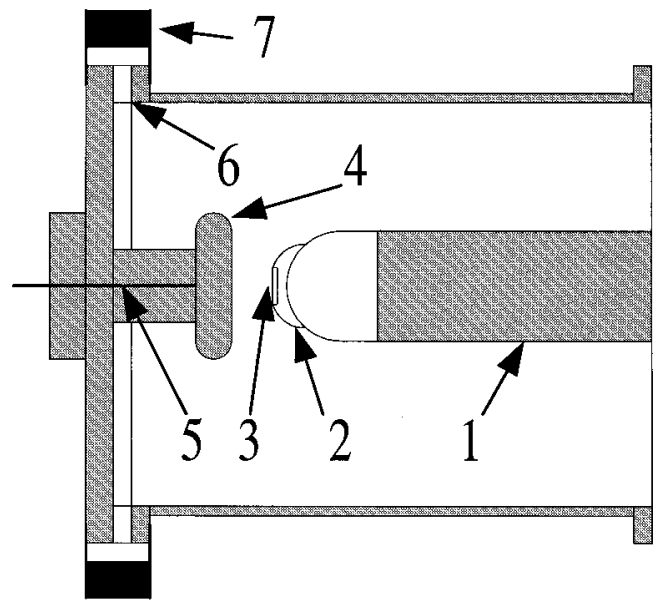

FIG. 1. Side view of MELBA anode-cathode gap. (1) Cathode stalk; glyptal insulating enamel extends $75 \mathrm{~cm}$ from the base of the stalk, the left 10 $\mathrm{cm}$ of the stalk are bare anodized aluminum so as to not be affected by the rf discharge. (2) Cathode assembly; 304 stainless steel cathode holder with T3-2011 aluminum cathode center insert. (3) Aluminum cathode insert. (4) Anode assembly; 304 stainless steel anode ring holder with Ti-6-4 center anode insert. (5) Radio frequency power antenna. (6) Insulating ring between anode plate and ground. (7) Copper jumper with Pearson coil to measure axial current; four total around anode.

ending at 20-30 s before the MELBA shot so that the fill gas could be pumped out to a base pressure low enough for MELBA to fire. During each MELBA shot, real-time data were acquired for voltage, diode current, axial current, hydrogen alpha optical emission $(656.28 \mathrm{~nm})$ and aluminum I optical emission $(396.15 \mathrm{~nm})$ at the cathode surface. Axial current is defined as current flowing from the cathode to the anode (from parts 2 and 3 to part 4 in Fig. 1), while diode current is defined as total current flowing through the cathode stalk, and includes the fraction of current that flows radially. The hydrogen and aluminum optical emission measurements were made with two monochromators with photomultiplier (PM) tubes tuned into the specific optical emission lines so that time-resolved measurements of the surface contaminant plasma (hydrogen) and bulk metal plasma (aluminum) could be made.

Four test protocols of rf plasma processing were used: dirty (no processing), argon (99.998\%) processing, argon/ oxygen mix $(90 \% / 10 \%)$ processing, and two-stage process-

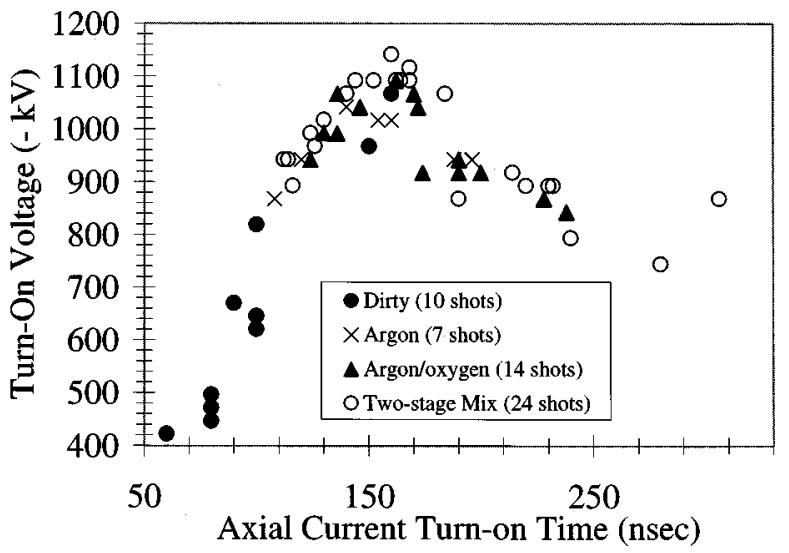

FIG. 2. Turn-on voltage vs turn-on time for a $4 \mathrm{~cm}$ MELBA A-K gap for each of the four test protocols. Time is measured from the start of the MELBA voltage pulse.
TABLE I. Summary of results for each of the four test cases. Number of shots taken for each case are: dirty (10), argon processed (7), argon/oxygen processed (14), two-stage mix processed (24).

\begin{tabular}{lcc}
\hline \hline Processing protocol & Turn-on voltage & Axial current turn-on time \\
\hline Dirty & $662 \pm 174 \mathrm{kV}$ & $100 \pm 22 \mathrm{~ns}$ \\
Argon & $967 \pm 49 \mathrm{kV}$ & $159 \pm 25 \mathrm{~ns}$ \\
Argon/oxygen & $974 \pm 67 \mathrm{kV}$ & $171 \pm 27 \mathrm{~ns}$ \\
Two-stage mix & $981 \pm 97 \mathrm{kV}$ & $175 \pm 42 \mathrm{~ns}$ \\
\hline \hline
\end{tabular}

ing (argon/oxygen followed by argon). The two-stage cleaning protocol utilized a low power reactive argon/oxygen discharge to remove surface hydrogen and leave a minimal oxide layer, followed by a higher power inert argon discharge to remove the oxide layer and surface particulate. A 5 min, $100 \mathrm{~W}, 13.56$ mTorr rf discharge of 90/10 argon/ oxygen followed by a $15 \mathrm{~min}, 200 \mathrm{~W}, 13.56 \mathrm{mTorr}$ rf discharge of $99.998 \%$ argon has given the best results to date. Processing plasma parameters measured by Langmuir probe and optical spectroscopy were in the range of $10^{11} \mathrm{~cm}^{-3}$ electron density and $\sim 1 \mathrm{eV}$ electron temperature.

Figure 2 is a graph of turn-on voltage required versus axial current emission turn-on time for a $4 \mathrm{~cm} \mathrm{A-K}$ gap for all four test protocols. The turn-on voltage required and turn-on time for axial electron emission were both increased with rf plasma processing, yielding the best results for the two-stage gas mix. Experimental results are summarized in Table I. The reason for the optimal increase in voltage from 662 to $981 \mathrm{kV}$ and delay in turn-on time from 100 to $175 \mathrm{~ns}$ is the removal of hydrogen and water vapor from the cathode surface as shown below in Fig. 3. Residual gas analysis (RGA) measurements have shown that the background vacuum of MELBA is $\sim 80 \%$ water vapor. At $10^{-6}$ Torr (normal operating pressures) many monolayers of water adsorb on vacuum surfaces. It is this layer of water vapor and surface contaminants that breaks down first and is the major source of anode and cathode plasma formation and parasitic current loss. ${ }^{7-10,12}$

Figure 3 shows oscillograms of voltage and PM tube time-resolved optical spectroscopy measurements of the

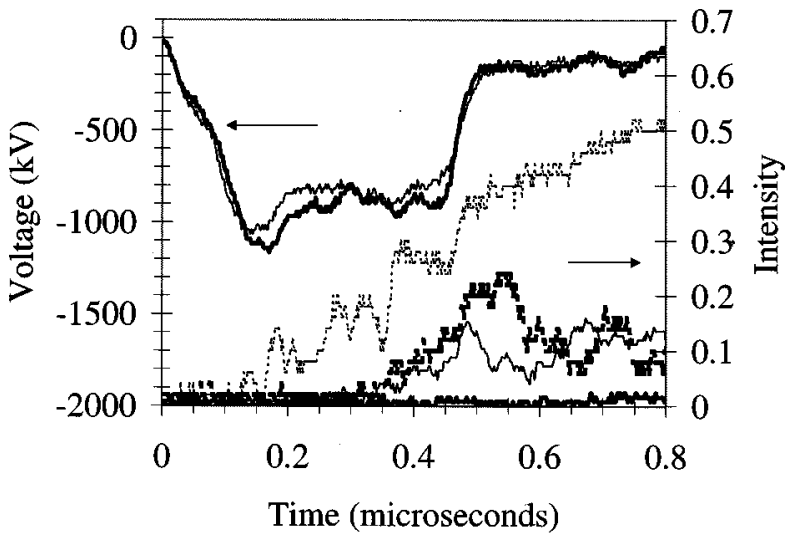

FIG. 3. Cathode voltage and optical spectroscopy measurements of the $656.28 \mathrm{~nm}$ hydrogen alpha line and $396.1 \mathrm{~nm}$ Al I line for a dirty vs twostage mix rf processed MELBA shot. The light solid trace is the dirty voltage/hydrogen MELBA shot, the bold solid trace is the rf processed voltage/hydrogen MELBA shot. The light dotted trace is the dirty aluminum MELBA shot, the bold dotted trace is the rf processed aluminum MELBA shot. Time starts at the beginning of the MELBA firing sequence. 


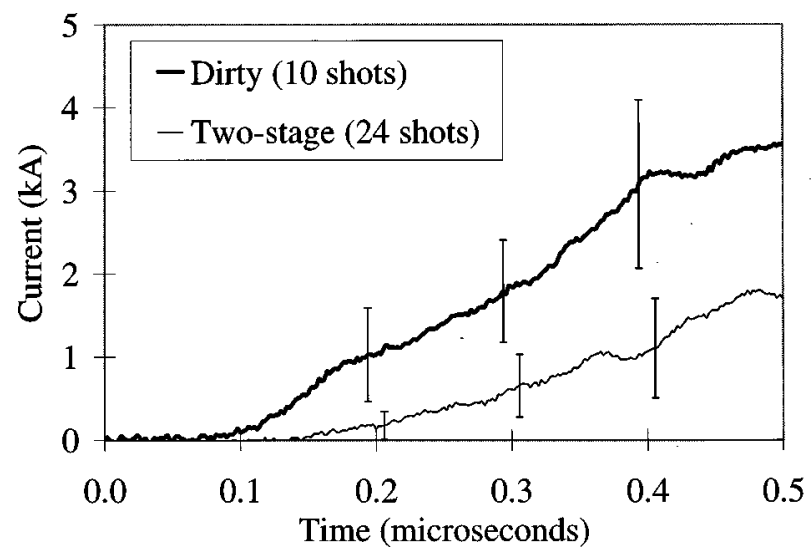

FIG. 4. Axial current emission for the dirty vs two-stage rf processed test cases. Time is measured from the start of the MELBA voltage pulse. Closure velocities ranged from 4.5 to $5.5 \mathrm{~cm} / \mu$ s for the dirty case and $3.5-4.5$ $\mathrm{cm} / \mu$ s for the two-stage rf processed case.

$656.28 \mathrm{~nm} \mathrm{H}$ alpha line and $396.1 \mathrm{~nm}$ aluminum I line for a dirty versus two-stage rf plasma processed MELBA shot. After plasma processing, the hydrogen optical emission is almost nonexistent and the aluminum emission has been reduced. The absence of hydrogen (from water vapor and surface contamination) on the cathode surface corresponds well with a reduction in the magnitude of axial current emission and delay in axial emission current turn on.

Figure 4 is a graph of axial current emission versus time for a $4 \mathrm{~cm}$ MELBA A-K gap for the dirty versus two-stage plasma protocols averaged over multiple shots. The difference in magnitude and slopes of axial current measured for dirty versus two-stage rf processed case correspond to a decrease in perveance and closure velocity for the two-stage rf processed protocol. The calculated plasma closure velocities ${ }^{1-3}$ for the dirty case were $4.5-5.5 \mathrm{~cm} / \mu \mathrm{s}$ vs $3.5-$ $4.5 \mathrm{~cm} / \mu$ s for the two-stage rf processed case.

Radio frequency plasma processing has been shown to reduce parasitic current loss due to surface contaminants, raise the voltage required to initiate electron emission, and delay the axial current turn-on time from an aluminum cathode on a MV electron beam accelerator. The two-stage reactive/inert rf processing protocol gave the highest voltage hold off and lowest emission current with the largest delay in aluminum plasma optical emission turn-on time. Correspondingly, hydrogen optical emission was removed with the two-stage protocol. Average macroscopic electric field strength required to initiate electron emission at the cathode was increased from $165 \pm 43$ to $245 \pm 24 \mathrm{kV} / \mathrm{cm}$.

This research was supported by DOE Contract No. DEAC04-94AL85000 to Sandia National Labs and Sandia Contract No. AL-7553 to the University of Michigan. The authors express their appreciation and thanks to Dr. Tom Mehlhorn for his continual support of JIR research. This research was performed under appointment to the Fusion Energy Sciences Fellowship program administered by the Oak Ridge Institute for Science and Education under Contract No. DEAC05-76OR00033 between the U.S. Department of Energy and the Oak Ridge Associated Universities.

${ }^{1}$ R. K. Parker, Ph.D. thesis, University of New Mexico, 1974.

${ }^{2}$ R. K. Parker, R. E. Anderson, and C. V. Duncan, J. Appl. Phys. 45, 2463 (1974).

${ }^{3}$ D. D. Hinshelwood, IEEE Trans. Plasma Sci. PS-11, 188 (1982).

${ }^{4}$ D. Hinshelwood, Ph.D. thesis, Massachusetts Institute of Technology, 1985.

${ }^{5}$ G. Niedermann, N. Sankarraman, R. J. Noer, and O. Fischer, J. Appl. Phys. 59, 892 (1986).

${ }^{6}$ M. E. Cuneo, Ph.D. thesis, University of Michigan, 1989.

${ }^{7}$ M. E. Cuneo, P. R. Menge, D. L. Hanson, W. E. Fowler, M. A. Bernard, G. R. Ziska, A. B. Filuk, J. E. Bailey, M. P. Desjarlais, T. R. Lockner, T. A. Mehlhorn, T. D. Pointon, S. A. Slutz, M. A. Stark, and R. A. Vesey, IEEE Trans. Plasma Sci. 25, 229 (1997).

${ }^{8}$ J. Halbritter, IEEE Trans. Electr. Insul. EI-18, 253 (1983).

${ }^{9}$ J. Halbritter, IEEE Trans. Electr. Insul. EI-20, 671 (1985).

${ }^{10}$ J. Halbritter, Appl. Phys. A: Solids Surf. 49, 49 (1986).

${ }^{11}$ R. V. Latham, High Voltage Vacuum Insulation (Academic, San Diego, 1995).

${ }^{12}$ P. R. Menge and M. E. Cuneo, IEEE Trans. Plasma Sci. 25, 252 (1997). 